\begin{tabular}{|c|c|c|}
\hline $4.8 g$ & $\begin{array}{c}\text { European Association for the } \\
\text { Development of Renewable Energies, Environment } \\
\text { and Power Quality (EA4EPQ) }\end{array}$ & $\begin{array}{l}\text { International Conference on Renewable Energies and Power Quality } \\
\text { (ICREPQ'12) } \\
\text { Santiago de Compostela (Spain), 28th to 30th March, } 2012\end{array}$ \\
\hline
\end{tabular}

\title{
Power output fluctuations in large PV plants
}

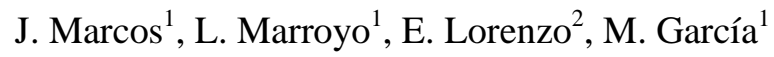 \\ ${ }^{1}$ Departamento de Ingeniería Eléctrica y Electrónica, \\ Edifico Los Pinos, Universidad Pública de Navarra, \\ Campus Arrosadía, 31006, Pamplona, Spain. javier.marcos@unavarra.es \\ ${ }^{2}$ Instituto de Energía Solar, \\ Grupo de Sistemas, ETSI Telecomunicación, \\ Ciudad Universitaria, s/n, 28040, Madrid, Spain
}

\begin{abstract}
The power generated by large photovoltaic (PV) plants is of an intermittent nature, due to cloud passage, which can create considerable fluctuations in output. Therefore, if the penetration of $\mathrm{PV}$ power in the overall electricity system is high enough, these fluctuations could have a serious effect on the quality and safety of the power supply. This article aims to characterise these fluctuations and to look at the smoothing effect of the PV plant size and that of the geographical dispersion of the PV plants.
\end{abstract}

\section{Key words:}

large PV plants; grid-connected; power fluctuations; PV plant model; geographical dispersion.

\section{Introduction}

The variations in irradiance produced by changes in cloud cover can cause rapid fluctuations in the power generated by large PV plants. For intervals of less than 10 minutes, these fluctuations are directly absorbed by the electricity system in the form of frequency variations, yet without the utility operator having the response capacity to correct the imbalances. If these frequency deviations exceed the permitted limits, then there is a risk of a power system failure. This fact, together with the high levels of penetration achieved by $\mathrm{PV}$ power generation sector over the last few years, alerted the grid operator in Spain, Red Eléctrica de España (REE), who was led to promote a research initiative to study these fluctuations. The Instituto de Energía Solar (IES), the INGEPER Research Group of the Universidad Pública de Navarra, and Acciona Solar also took part in the project.

This paper presents the main conclusions reached in the project. The results are shown for the synchronised continuous monitoring, with a one second resolution, from April 2008 onwards, of 7 PV plants located in Spain and with output powers ranging from $1 \mathrm{MWp}$ to $9.5 \mathrm{MWp}$ (a total of 20 MWp). The research focussed on studying the irradiance and power fluctuations for time intervals of less than 10 minutes, paying particular attention to the characterisation of the magnitude and frequency of occurrence of these fluctuations. The study also analysed the influence of the PV plant size and the number of PV plants grouped in a multi-site system, on the magnitude of the fluctuations. Finally, a PV plant was modelled, making it possible to simulate the dynamics of the power generated by a PV plant based on irradiance data.

\section{Experimental set-up}

A review of the scientific literature prior to this study reveals a significant lack of experimental data on the magnitude of fluctuations. The number of studies on the subject is significantly small and it has only been over the last few years when the study of the fluctuations has gradually taken on greater importance. In this way, irradiance fluctuations have been monitored and analysed in countries such as Germany [1], Japan [2], Belgium [3] and Australia [4]. On the other hand, as far as the study of power fluctuations is concerned, the number of studies made is even more limited. The literature available only includes measurements with a 5 minute resolution on $100 \mathrm{PV}$ systems (243 kWp in total) in Germany [5]; data taken at 10 second and 1 minute intervals at a fixed 4.6 MWp system and measurements taken every 10 minutes at three $100 \mathrm{kWp}$ plants in Arizona (USA) [6] and, finally, data recorded with a one minute resolution over a 3 month period on $52 \mathrm{PV}$ systems in Japan, with an approximate mean power per system of $3.2 \mathrm{kWp}[7]$.

In an attempt to cover this lack of prior empirical research, within the framework of this project, monitoring equipment has been designed, 
programmed and installed in a number of PV plants owned by Acciona Solar. This equipment, which has been operating since April 2008, makes a synchronised recording every second of the irradiance and the power generated by $7 \mathrm{PV}$ plants located in Spain. The plants have powers ranging from $1 \mathrm{MWp}$ to $9.5 \mathrm{MWp}$ (a total of $20 \mathrm{MWp}$ ). Likewise, data are available from two sections of the same plant (48 $\mathrm{kWp}$ and $143 \mathrm{kWp}$ ), also recorded with a one second resolution. Six of the plants are located in the South of Navarre, separated by distances ranging from $6 \mathrm{~km}$ to $60 \mathrm{~km}$. The seventh plant is situated in the locality of Socuéllamos (Ciudad Real) at a distance of $345 \mathrm{kms}$ from the other plants in Navarre (Fig 1). The most representative details of these plants are shown in the following table:

\begin{tabular}{|c|c|c|c|}
\hline PV plants & $\begin{array}{c}\text { Peak Power } \\
(\mathbf{k W p})\end{array}$ & $\begin{array}{c}\text { Transformer Power } \\
(\mathbf{k W}), \boldsymbol{P}^{*}\end{array}$ & $\begin{array}{c}\text { Area } \\
(\mathbf{H a}), \boldsymbol{S}\end{array}$ \\
\hline Arguedas & 958 & 775 & 4,1 \\
\hline Sesma & 990 & 800 & 4,2 \\
\hline Cintruénigo & 1438 & 1155 & 6,4 \\
\hline Rada & 1780 & 1400 & 8,7 \\
\hline Castejón & 2640 & 2.000 & 11,8 \\
\hline Milagro & 9500 & 7243 & 52 \\
\hline Socuéllamos & 2600 & 1975 & 18 \\
\hline Total & 19906 & 15348 & - \\
\hline
\end{tabular}

Table 1: Characteristics of the photovoltaic (PV) plants.
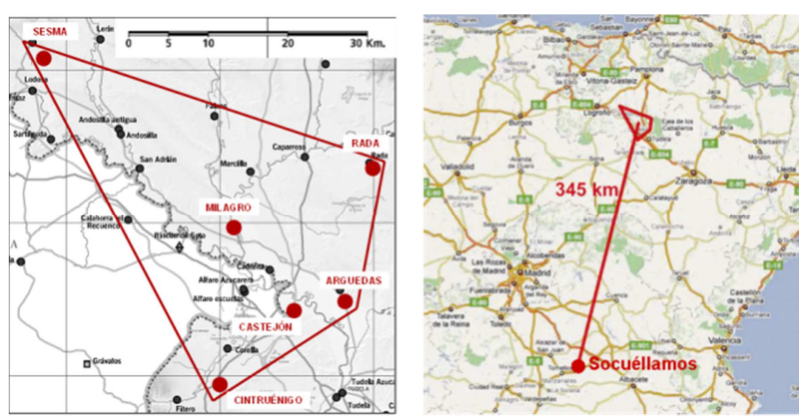

Fig. 1: Location of the 7 photovoltaic (PV) plants studied.

\section{Fluctuation definition}

The magnitude of a power fluctuation $\Delta P_{\Delta t}(t)$ at an instant $t$ for a given sampling period, $\Delta t$, is calculated as the difference between the two power outputs, normalized to the transformer power $P^{*}$ of the plant under consideration, Eq. (1). That is:

$$
\Delta P_{\Delta t}(t)=\frac{[P(t+\Delta t)-P(t)]}{P^{*}}
$$

This definition can also be applied to irradiance fluctuations, $\Delta G_{\Delta t}$, normalizing by $G^{*}=1000 \mathrm{~W} / \mathrm{m}^{2}$.

\section{Fluctuation at a PV plant}

Fig. 2 shows the irradiance fluctuation distributions observed at the Cintruénigo site over the course of a year (from May 2008 to April 2009) for $\Delta t$ equal to $10,20,60$ and $600 \mathrm{~s}$. All the distributions are normalized, so that the total area below the curve is equal to one. Visual appearance gives a different impression because the frequency axis has a logarithmic scale. A positive correlation between the fluctuation magnitude and the sampling time is observed again. Table 2 shows the values of some fluctuation intervals. Note that a significant fluctuation of, let us say, more than $3 \%$ in $1 \mathrm{~s}$, is relatively rare. In fact, this is why frequency is presented in logarithmic scale in Fig. 6. However, the relative frequency is close to $41 \%$ at $600 \mathrm{~s}$.

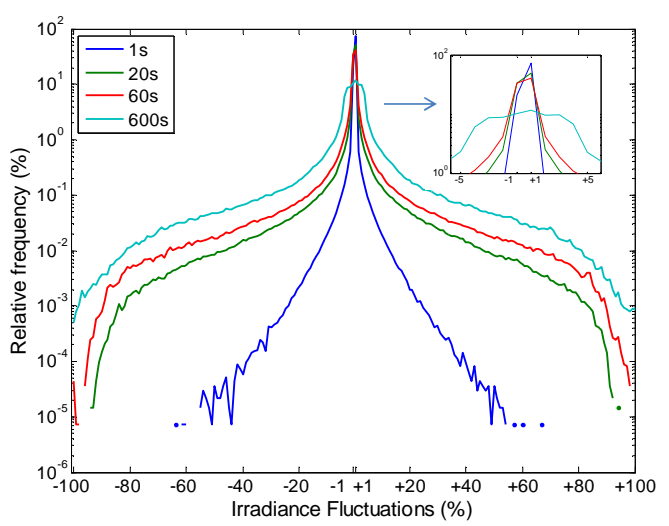

Fig. 2: Distributions of the irradiance fluctuations, $\Delta \mathrm{G} \Delta \mathrm{t}(\mathrm{t})$, recorded at the Cintruéingo site in the course of a year, for $\Delta \mathrm{t}=10,20,60$ and $600 \mathrm{~s}$.

It is noteworthy that all the irradiance fluctuation features calculated at the rest of places (and not presented here), essentially coincide with the features at Cintruénigo site, in accordance with the fact that all sites belong to the same climatic region. Consequently, the power fluctuations will not be affected by the effects of geographic location.

\begin{tabular}{|c|c|c|c|c|}
\hline \multirow{2}{*}{$\Delta \mathbf{G}(\%)$} & \multicolumn{4}{|c|}{$\Delta t(s)$} \\
\hline & $1 \mathrm{~s}$ & $20 \mathrm{~s}$ & $60 \mathrm{~s}$ & $600 \mathrm{~s}$ \\
\hline $0 \% \leq \Delta \mathrm{G} \leq 3 \%$ & $98.94 \%$ & $92.51 \%$ & $87.50 \%$ & $59.37 \%$ \\
\hline $3 \% \leq \Delta G \leq 10 \%$ & $0.92 \%$ & $4.47 \%$ & $7.61 \%$ & $28.24 \%$ \\
\hline $10 \% \leq \Delta \mathbf{G} \leq \mathbf{5 0 \%}$ & $0.16 \%$ & $2.71 \%$ & $4.66 \%$ & $11.25 \%$ \\
\hline $50 \% \leq \Delta G \leq 100 \%$ & $2.35 \cdot 10^{-4} \%$ & $0.32 \%$ & $0.70 \%$ & $1.35 \%$ \\
\hline
\end{tabular}

Table 2: Influence of $\Delta t$ on the frequency distributions of the fluctuations.

On the other hand, Fig. 3 shows the maximum irradiance fluctuations recorded at the various sites for an entire year in relation to the different sampling times $\Delta t$ considered. The value of the maximum fluctuation is extremely useful for the power utility operator, making it possible to check the robustness of a grid. The values observed for $\Delta t$ greater than 2 seconds are over $80 \%$. Therefore, although the 
irradiance fluctuations can reach very high values, this is a relatively rare phenomenon in annual terms.

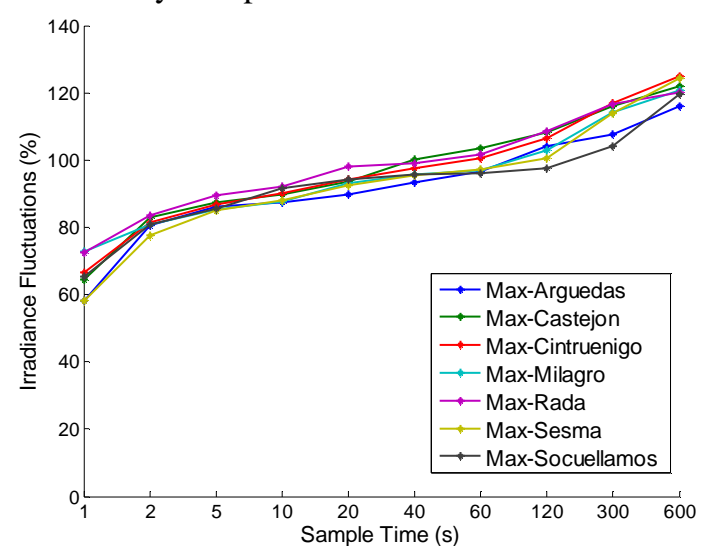

Fig. 3: Maximum irradiance fluctuation recorded at each PV plant site vs. $\Delta t$.

With regard to the power fluctuations produced by a PV plant, the magnitude of these fluctuations is influenced not only by $\Delta t$, but also by the PV plant size. It is therefore to be expected that, the larger the plant size, the lower the power fluctuations in relation to the irradiance fluctuations. This reasoning is based on the fact that a cloud will always take longer to cover an entire PV plant than an irradiance measurement module. Thus, the shorter the sampling time, the greater the smoothing effect. This fact can clearly be observed in Fig. 4, showing the annual power fluctuation distributions for the powers recorded at each PV plant for $\Delta t=20 \mathrm{~s}$. As was to be expected, the filtering effect on the fluctuations increases in line with the increased plant size whilst, on the other hand, it decreases as $\Delta t$ increases. Likewise, this smoothing effect can be clearly seen in Fig. 5, showing the value of the maximum power fluctuation recorded over a year at each plant in relation to $\Delta t$. However, for $\Delta t=600 \mathrm{~s}$, the smoothing effect is non-existent, and fluctuations of $90 \%$ can be reached, regardless of the plant size.

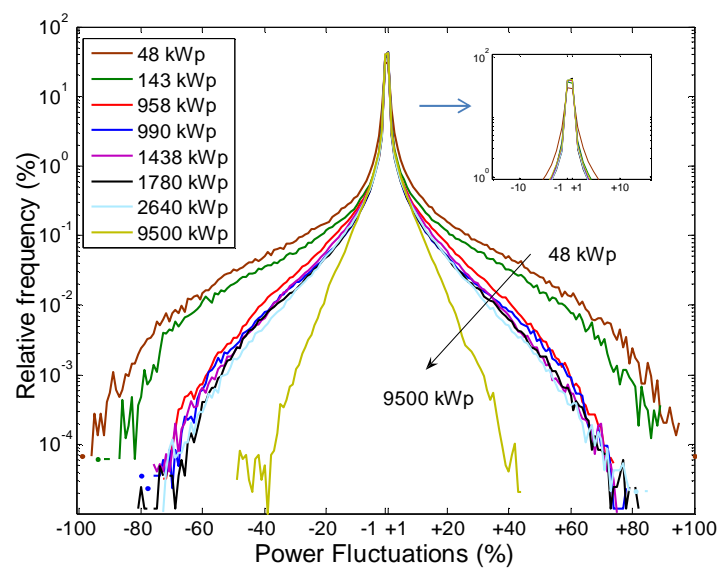

Fig. 4: Distributions of the power fluctuations, $\Delta P_{\Delta t}(t)$, recorded at each PV plant over an entire year, for $\Delta t=20 \mathrm{~s}$.

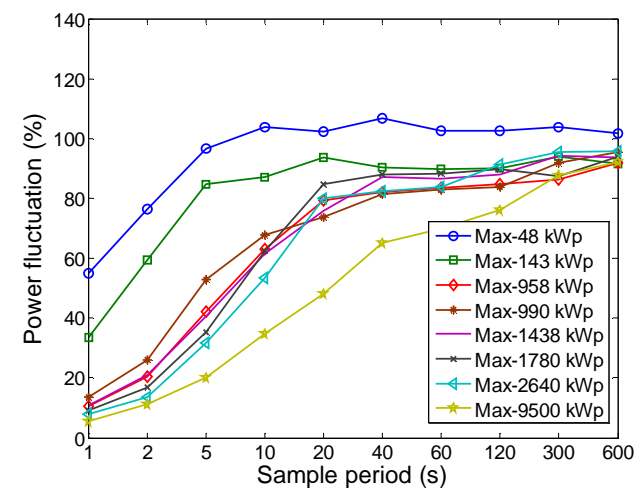

Fig. 5: Maximum power fluctuations recorded at each PV plant vs. $\Delta t$.

The relationship between thee smoothing effect and PV plant size is embodied in the following proposed empirical Eq. (2), which determines the peak power fluctuation value of a PV plant of any size $S[\mathrm{Ha}]$ and for any sampling time $\Delta t$ :

$$
\operatorname{Max}\left(\Delta P_{\Delta t}\right)=90 \% \cdot\left(1-e^{-0.24 \cdot \Delta t}\right) \cdot S^{-c}
$$

Smoothing coefficient $c$ for $\Delta t=1 \mathrm{~s}$ is 0.5 , thus the smoothing effect on the fluctuations can be described quite accurately by a $1 / \mathrm{N} S$ law. However, for $\Delta t=600 \mathrm{~s}$, coefficient $c$ is equal to 0.02 which supports the idea that, for these sampling times, the plant size is not a smoothing factor for the fluctuations. A more detailed and supplementary account of the results shown in this and the previous section can be found in [8].

\section{Fluctuations in a multi-site PV plant system}

We shall now go on to quantify the smoothing of PV power fluctuations by geographical dispersion. Thanks to the synchronised monitoring made, it is possible to determine, second by second, the combined power generated by a group of PV plants, by monitoring plant behaviour, in the same way as the system operator.

Fig. 6 shows the normalised distributions for the fluctuations recorded in 2009 for $\Delta t=60 \mathrm{~s}$ corresponding to just one plant (Arguedas, $P_{I}^{*}=0.775 \mathrm{MW}$ ), to a group of 3 plants (Castejón, Milagro and Sesma, $\left.P_{3} *=10.043 \mathrm{MW}, \mathrm{COMB} 3\right)$ and a further group of 6 plants (all located in southern Navarre, $\left.P_{6}^{*}=13,373 \mathrm{MW}, \mathrm{COMB} 6\right)$. It can clearly be observed how the magnitude and frequency of the fluctuations decreases in line with an increase in the number of plants grouped together, $N$. It is important to emphasise the fact that the smoothing effect extends as far as the long sampling times $(\Delta t=600 \mathrm{~s})$ in contrast to the effect observed for plant size. 


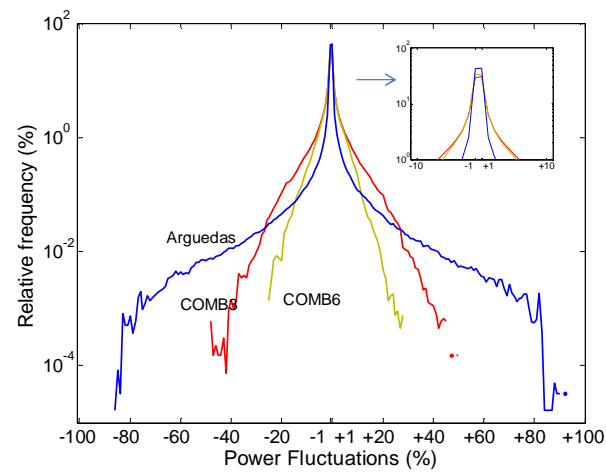

Fig. 6: Distributions of the power fluctuations $\Delta P_{\Delta t, N}$, recorded in the course of an entire year (2009) at the Arguedas PV plant and the combination of $N=3$ (COMB3) and $N=6$ (COMB6) for multi-site PV plants, all in Navarre, for $\Delta t=60 \mathrm{~s}$.

Fig. 7 below shows the value of the peak power fluctuation recorded versus the sampling time $\Delta t$ based on the number of plants grouped together, $N$, for all plants located in Navarre. It is possible to observe a considerable reduction in the magnitude of the fluctuations, irrespective of the $\Delta t$. Furthermore, it was found that for a given $N$, no plant combination had a greater smoothing effect than another. In other words, the location of each PV plant in relation to the others within the group, had no effect on the magnitude of the smoothing effect. Therefore, the fluctuations of a group of PV plants will be smoothed provided that there is sufficient distance from one plant to another. This leads on to the question of what is the minimum distance between plants in order to ensure that the plant fluctuations remain independent of each other and to guarantee smoothing. For this, Fig. 8 shows the distribution of fluctuations for the entire year of 2009 for the Arguedas-Castejón group, separated by a distance of $6 \mathrm{kms}$, and the distribution for the Arguedas-Socuéllamos pair, $345 \mathrm{kms}$ apart. As can be seen, there are hardly any differences between the fluctuations of any two-plant combinations. Therefore, a distance of $6 \mathrm{kms}$, being the shortest distance between any plant and our observatories (located at Arguedas and Castejón) is sufficient to guarantee the independence of plant power fluctuations for time intervals of less than 10 minutes or, to put it another way, to guarantee smoothing by geographical dispersion.

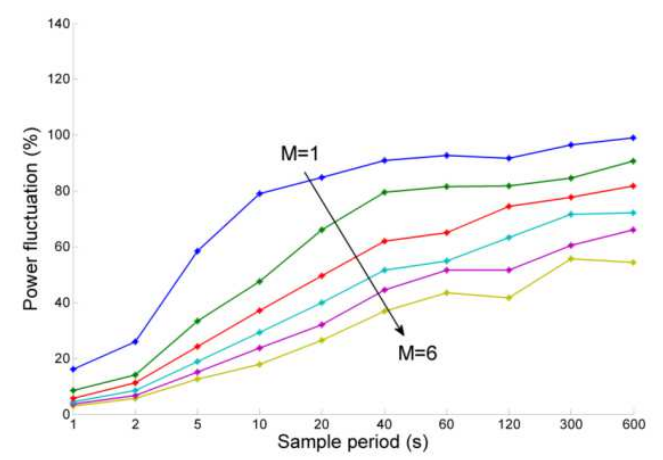

Fig. 7: Peak power fluctuations $\Delta P_{\Delta t, N}$, recorded over an entire year for all possible combinations for $N=1 \ldots 6$ plants (all in Navarre).

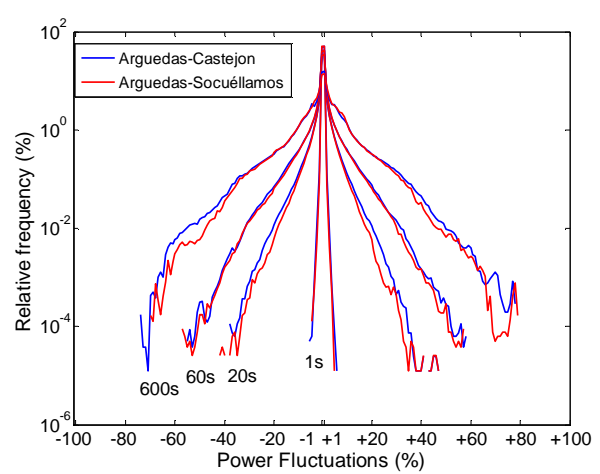

Fig. 8: Distribution of the power fluctuations $\Delta P \Delta t, 2$, recorded over an entire year (2009) at the combination of Arguedas-Castejón PV plants (distance $=6 \mathrm{~km}$ ) and Arguedas-Socuéllamos $($ distance $=345 \mathrm{~km})$ for $\Delta t=1,20$, 60 , and $600 \mathrm{~s}$.

The data shown in Fig. 7 was used to obtain an empirical Eq. (3) which provides the value of the peak power fluctuation for a group of $N$ PV plants of a certain size $S$, for any $\Delta t$ of less than 10 minutes.

$$
\begin{gathered}
99^{t h}\left(\Delta P_{\Delta t, N}\right)=99^{t h}\left(\Delta P_{600,1}\right) \cdot\left(1-e^{-0.24 \cdot \Delta t}\right) \cdot S^{-c} \cdot N^{-a} ; \\
a, c>0
\end{gathered}
$$

This equation describes the smoothing of the fluctuations resulting from the plant size and also the geographical dispersion. Whenever coefficients $a$ are greater than $c$, as in Table 4 , the smoothing due to dispersion is greater than that due to plant size.

\begin{tabular}{|c|c|c|}
\hline $\boldsymbol{\Delta} \boldsymbol{t}$ & $\mathbf{c}$ & $\mathbf{a}$ \\
\hline $\mathbf{1 s}$ & 0.49 & 25.55 \\
\hline $\mathbf{5 s}$ & 0.29 & 64.09 \\
\hline $\mathbf{2 0 s}$ & 0.11 & 88.2 \\
\hline $\mathbf{6 0 s}$ & 0.05 & 91.6 \\
\hline $\mathbf{6 0 0} \mathbf{s}$ & 0.02 & 94.07 \\
\hline
\end{tabular}

Table 4: Estimated parameters $a$ and $c$ for empirical Eq. (3).

Based on Eq. (3), Fig. 9 shows the fluctuations of a total PV power of $100 \mathrm{MW}$ in relation to the number of plants amongst how this power is distributed and sampling time $\Delta t$. As can be seen, after approximately 100 plants (1 MW each) the fluctuations are smoothed considerably, and it is debatable whether the power needs to be distributed still further, given the fact that this would have little effect on the smoothing achieved. All these conclusions, together with some additional analyses can be found in [9].

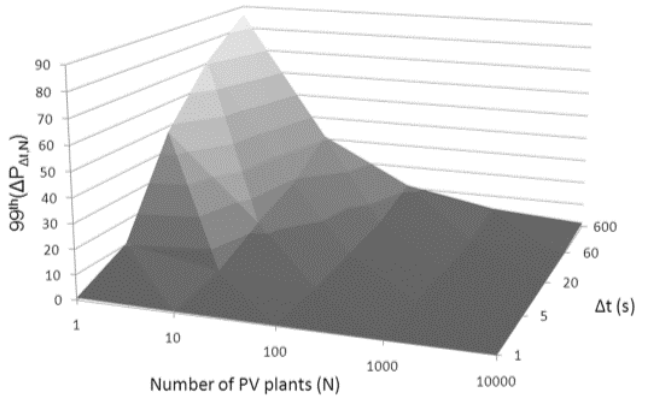

Fig. 9: Estimated $\operatorname{Max}\left(\Delta P_{\Delta t, N}\right)$ values for different $\Delta t$ and $N$, $P^{*} \mathrm{PV}$ plants combinations; 


\section{Fluctuations in the frequency domain}

The periodicity character of irradiance and the power generated by a PV plant due to solar resource, invites an analysis of these two variables in the frequency domain. The study of the PV power fluctuations in this domain makes it possible to consider the plants as low-pass filters. Indeed, what has up to now been called smoothing due to plant size, could be interpreted as a filtering of the signal representing irradiance. The Discrete Fourier Transform was used to convert to the frequency domain.

Fig. 10 shows the spectrum for the irradiance normalised by $G^{*}$, recorded for the entire year of 2009 at the Milagro plant, together with the normalised power spectra for this same plant $\left(P^{*}=9.5 \mathrm{MWp}\right)$ and that for the Sesma plant $\left(P^{*}=0.9 \mathrm{MWp}\right)$, also recorded over this same period.

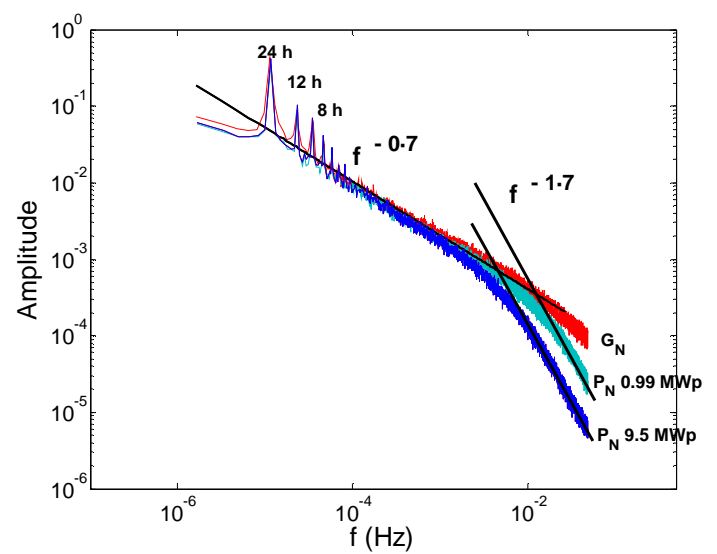

Fig. 10: Spectrum of the irradiance $G_{N}$ recorded at Milagro, output power $P_{N}$ at Sesma $\left(P^{*}=0.99 \mathrm{MW}\right)$ and Milagro $\left(P^{*}=9.5 \mathrm{MW}\right)$ over 1 year. The linear region for the higher frequencies of the power spectra can be well fitted by a function of the form $f^{-1.7}$

It is possible to observe the corresponding peaks at frequencies of $24,12 \mathrm{~h}$ etc, due to the cyclic nature of the solar resource, being independent of the spectrum considered. In the low frequency zone (up to approximately $10^{-3} \mathrm{~Hz}$ ), all the spectra had a similar performance, with a downward trend $\mathrm{f}^{-0.7}$. However, after a certain frequency $f_{c}$, which differed from one plant to another, a change in trend was observed, implying the increased smoothing of the higher frequencies. As was to be expected, the greatest smoothing was obtained for the plant with the largest size. The mathematical expression of the new trend is form $\mathrm{f}^{-1.7}$ and is independent of the PV plant considered. The cut-off point for functions $\mathrm{f}^{-0.7}$ and $\mathrm{f}^{-1.7}$ can be identified as cut-off frequency $f_{c}$ for the systems in question. Furthermore, the difference between the slope for the two functions is the unit, which means that the corresponding low pass filter is first order, with a pole at cut-off frequency $f_{c}$. This exercise was extended to the other PV plants, obtaining the corresponding value for $f_{c}$. These experimental values were generalised to obtain empirical Eq. (4) to provide the value of the cut-off frequency in relation to the area $S$ of a PV plant:

$$
f_{c}=0.021 \cdot S^{-0.5}
$$

As a result, it is now possible to propose a PV plant model relating the irradiance received in the tracker plane and the power generated, Fig. 11:

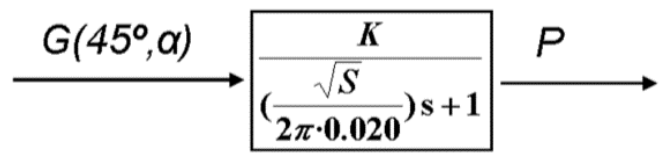

Fig. 11: Proposed transfer function for a PV plant with a surface area equal to $S$.

where $K$ is the energy gain of the PV plant $\left[\mathrm{m}^{2}\right]$. This model has been validated in both the time and frequency domains, thereby permitting the simulation of fluctuations in any real electricity system, insofar as it makes it possible to convert relatively easy-togenerate irradiance time data into power data for any potential PV plant size. By way of example, Fig. 12 shows the distributions of the real and simulated maximum daily fluctuations, for $\Delta t$ equal to $600 \mathrm{~s}$ at Milagro PV plant. As can be seen, the frequency distributions are very similar. A more detailed description of this model can be found in [10].

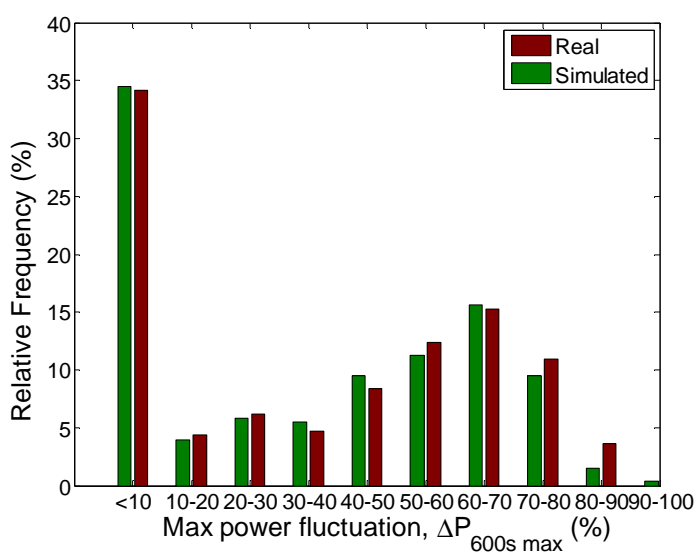

Fig. 12: Histograms of the real maximum daily power fluctuation observed in the course of a year at Milagro PV plant and the simulated fluctuations for $\Delta t=600 \mathrm{~s}$.

\section{Conclusions}

The quality and safety of the electricity supply can be seriously affected by large PV plant power fluctuations caused by the variability in irradiance due to cloud passage. This article has presented the study of these fluctuations, obtaining the following results:

- A unique monitoring system, in place since April 2008 , and which makes synchronous recordings with a one second resolution, of the power delivered to the grid by seven PV plants, with powers ranging from 1 MWp to $9.5 \mathrm{MWp}$ and with a total combined power of almost $20 \mathrm{MWp}$. 
- The power fluctuations occurring in a PV plant within time intervals of less than ten minutes, are very high and relatively infrequent, although they are smoothed in relation to the irradiance variations. The smoothing factor is the PV plant size, and is relevant for times of just a few seconds, disappearing as the sampling time increases. Likewise, an empirical equation is proposed, and which provides the value of the peak power fluctuation in relation to the PV plant size.

- The geographical dispersion of the PV plants is a highly effective way of smoothing the power fluctuations, even for ten minute sampling intervals. It is sufficient to locate two PV plants at a distance of 6 $\mathrm{kms}$, one from the other, to ensure that the fluctuations over 10 minute intervals are independent of each other and are smoothed out when combined. Furthermore, an empirical equation is proposed, that will give the value of the peak power fluctuation, based on the number of plants grouped together and the PV plant size.

- A spectral analysis of the power and irradiance data measured over the course of a year has made it possible to characterise a PV plant as a first order system and, specifically, as a low pass filter whose cut-off frequency is directly related to the plant size. This model can be a highly useful tool for utility operators, in the sense that the operator simply needs to obtain irradiance data and the PV plant size in order to simulate any photovoltaic scenario.

With regard to future lines of investigation, the conclusions of this work suggest the need to continue studying the correlation of fluctuations for distances of less than $6 \mathrm{~km}$. Currently ongoing and still provisional studies suggest that risky days result from the combination of high wind speed (about $60 \mathrm{~km} / \mathrm{h}$, or more) with intermediate daily clearness index $\left(0.35<K_{t}<0.65\right)$. Because wind speed and clearness index can be predicted from 1 day to another (in fact, it is already being done in standard weather forecast), this likely opens the door for future prediction of fluctuations in daily terms. Finally, there is a need to study the combination of PV generators - storage batteries. As well as limiting power fluctuations, this alternative would allow the PV systems to play an active part in frequency regulation and would also make it possible for the plants to increase their daily production curve, to a certain extent, in an endeavour to adapt it to consumption.

\section{Acknowledgements}

The authors would like to thank Red Eléctrica de España (REE) for its financial support and Acciona Solar for authorizing measurements at its PV plants and also for the helpful collaboration of its staff. This work has been also supported by the Spanish Ministry of Education and Science (grant number: DPI200914713-C03-01).

\section{References}

[1] Beyer HG, Decker B, Luther J, Steinberg-Willms R. "Spatial and temporal characteristics of short term fluctuations in solar radiation for PV-plant applications", 10th European Photovoltaic Solar Energy Conference, 8-12 April, 1991 Lisbon, Portugal, 453456.

[2] Otani K, Minowa J, Kurokawa K. Study on a real solar irradiance for analyzing a really-totalized PV systems. Solar Energy Materials and Solar Cells 1997; 47: 281288.

[3] Woyte A, Thong VV, Belmans R, Nijs J. Voltage fluctuations on distribution level introduced by photovoltaic systems. IEEE Transactions on Energy Conversion 2006; 21: 202-209.

[4] Woyte A, Belmans R, Nijs J. Fluctuations in instantaneous clearness index: analysis and Statistics. Solar Energy 2007; 81: 195-206.

[5] Wiemken E, Beyer HG, Heydenreich W, Kiefer K. Power characteristics of PV ensembles: experiences from the combined power production of 100 Grid connected PV systems distributed over the area of Germany. Solar Energy 2001; 70(6): 513-518.

[6] Curtright E, Apt J. The character of power output from utility-scale photovoltaic systems. Progress in Photovoltaic: Research and Applications 2008; 16: 241247.

[7] Murata A, Yamaguchi H, Otani K. A method of estimating the output fluctuation of many photovoltaic power generation systems dispersed in a wide area. ElectricalEngineering in Japan 2009; 166(4): 645-652.

[8] Marcos, J., Marroyo, L., Lorenzo, E., Alvira, D. and Izco, E. (2011), Power output fluctuations in large scale PV plants: One year observations with one second resolution and a derived analytic model. Progress in Photovoltaics: Research and Applications, Volume 19, issue 2, pages 218-227, March 2011. doi: 10.1002/pip.1016.

[9] Marcos, J., Marroyo, L., Lorenzo, E., Alvira, D. and Izco, E. (2011), PV power fluctuations smoothing by geographical dispersion. Progress in Photovoltaics: Research and Applications, Published online in Wiley Online Library (wileyonlinelibrary.com). doi: 10.1002/pip.1127.

[10]Marcos, J., Marroyo, L., Lorenzo, E., Alvira, D. and Izco, E. (2011), From irradiance to output power fluctuations: the PV plant as a low pass filter. Progress in Photovoltaics: Research and Applications, Volume 19, issue 5, pages 505-510, August 201. doi: 10.1002/pip.1063. 